\title{
Modelos alométricos na determinação da área foliar de Bauhinia monandra Kurz
}

\author{
Edilson Romais Schmildt*, Omar Schmildt, Rodrigo Sobreira Alexandre, \\ Adriano Alves Fernandes, Marcio Paulo Czepak
}

Universidade Federal do Espírito Santo, São Mateus, ES, Brasil

*Autor correspondente, e-mail: e.romais.s@gmail.com

\begin{abstract}
Resumo
O objetivo deste estudo foi avaliar a eficiência do ajuste de modelos matemáticos, para determinação da área foliar de Bauhinia monandra, utilizando o comprimento e/ou a largura das folhas como variáveis independentes. Foram analisadas folhas de plantas de três anos de idade para estimativa de equações nos modelos linear, quadrático e potencial. A validação a partir da área foliar estimada pelos modelos em função da área foliar observada mostrou que o melhor ajuste é para o modelo linear em função do produto entre comprimento e maior largura do limbo foliar; no entanto, a determinação da área foliar pode ser representada pelo uso apenas do comprimento ou da largura das folhas, com pouca perda de precisão. A representação que estima melhor a área foliar de Bauhinia monandra com maior simplicidade para aplicação é pelo modelo potencial $\hat{y}_{i}=1,0332 x_{i}^{19191}$ em que $x_{i}$ representa o comprimento de um dos lóbulos foliares simétricos.
\end{abstract}

Palavras-chave: análise de regressão, dimensão foliar, método não destrutivo, modelos matemáticos.

\section{Allometric models for Bauhinia monandra Kurz leaf area determination}

\begin{abstract}
The aim of this study was to evaluate the efficiency of the adjustment of mathematical models for determining Bauhinia monandra leaf area using the length and/or width of the leaves as independent variables. Leaves from plants with three years were used to the estimative of equations in linear, quadratic and potential models. The validation from the estimated leaf area as a function of the observed leaf area showed that the linear model based on the product of length and width of the largest leaf surface is the model that best fits. However, the leaf area determination can be represented by using only the length or width of the leaves with little loss of accuracy. A representation that better estimates Bauhinia monandra leaf area with easy application is the potential model $\hat{Y}_{i}=1.0332 x_{i}^{1.9191}$ in which xi represents the length of one of the symmetrical leaf lobes.
\end{abstract}

Keywords: regression analysis, leaf dimension, non-destructive method, mathematical models. 


\section{Introdução}

Bauhinia monandra Kurz, conhecida como "pata de vaca" é uma das 300 espécies do gênero Bauhinia (Vaz \& Tozzi, 2005), sendo utilizada com fins ornamental, forrageiro e medicinal.

Lusa \& Bona (2009) salientam que, apesar do amplo uso medicinal de folhas de diversas espécies de "pata de vaca", a composição fitoquímica é consideravelmente distinta entre as espécies, e consequentemente, os seus efeitos terapêuticos, necessitando de informações morfoanatômicas que caracterizem as espécies do gênero Bauhinia. Devido à sua relação direta com a fotossíntese, a área foliar é determinante em processos relacionados ao metabolismo, acúmulo de biomassa, fenologia e rendimento dos cultivos (Demirsoy, 2009).

As técnicas utilizadas para determinar a área do limbo foliar devem ser simples, rápidas, precisas (Souza et al., 2012) e de preferência que não destruam as folhas (Sarker et al., 2013). Esta determinação pode ser feita pelo uso de planímetros portáteis ou por equações previamente estabelecidas por estudos de modelagem matemática da área foliar (Demirsoy, 2009).

A modelagem estatística a partir de medidas alométricas de comprimento, largura e produto entre os dois tem sido investigada para auxiliar nos estudos de crescimento de plantas em diversas espécies como mangueira (Lima et al., 2012), amendoim (Cardozo et al., 2014) e abacaxizeiro (Francisco et al., 2014). No entanto, não se encontrou na literatura trabalhos que façam a modelagem por método não destrutivo em Bauhinia monandra. Assim, o objetivo deste estudo foi avaliar a eficiência do ajuste de modelos matemáticos, para determinação da área foliar de Bauhinia monandra.

\section{Material e Métodos}

As folhas foram coletadas em cinco plantas adultas (40 folhas em cada planta), oriundas de propagação seminífera, com idade de três anos, em área rural, no município de Linhares, com Latitude $19^{\circ} 11^{\prime} 13^{\prime \prime}$ S, Longitude $40^{\circ} 05^{\prime} 39^{\prime \prime} \mathrm{O}$ e altitude de $30 \mathrm{~m}$.

Foram avaliadas apenas folhas inteiras, sem sintomas de ataque de pragas e doenças, que foram colhidas, acondicionadas em sacos plásticos, e transferidas para o laboratório de Melhoramento de Plantas da Universidade Federal do Espírito Santo, onde as medidas foram realizadas. Foram feitas as medições de cada folha relativas ao comprimento de um dos lóbulos simétricos do limbo (C) e da maior largura (L). Com os dados de C e de L, determinou-se também o produto entre $\mathrm{C}$ e $\mathrm{L}(\mathrm{CL})$. Para todas as folhas coletadas foram computadas a área foliar observada (AFO) que foi determinada por método de imagens digitais, obtidas em scanner HP Deskjet $F 4480^{\circledR}$, em superfície contrastante de coloração branca. Para a medida direta da área foliar as imagens digitais foram manuseadas utilizando o Software Image $J^{\circledR}$ (Collins, 2007).

A validação foi feita com base nos valores estimados pelo modelo $\left(\hat{Y}_{\mathrm{i}}\right)$ e os valores observados $\left(Y_{i}\right)$ em 55 folhas colhidas aleatoriamente nas mesmas cinco árvores, dois meses após a coleta das folhas para modelagem.

Verificou-se a adequação do uso conjunto de folhas das cinco plantas, realizandose análise de variância para C, L, CxL, AFO e índice de correção ( $f=A F O / C X L)$. Foi utilizado o modelo matemático: $Y_{i j}=m+p_{i}+f_{i j}$ em que $Y_{i j}$ = valor da variável observado na i-ésima planta $(i=1,2, \ldots, 5)$ da j-ésima folha $(j=1,2, \ldots, 40) ; m=$ constante; $\mathrm{p}_{\mathrm{i}}=$ efeito aleatório da planta $\mathrm{i} ; \mathrm{e}, \mathrm{f}_{\mathrm{ij}}=$ efeito aleatório de folhas.

$$
\text { Para modelar a AFO }
$$

(variável dependente $=Y_{\mathrm{i}}$ ) em função das medidas alométricas como variáveis independentes $\left(\mathrm{x}_{\mathrm{i}}\right)$, utilizou-se os modelos linear $\left(Y_{i}=\beta_{0}+\beta_{1} x_{i}+e_{i}\right)$, quadrático $\left(Y_{i}=\beta_{0}+\beta_{1} x_{i}+\beta_{2} x_{i}^{2}+e_{i}\right)$ e potência $\left(Y_{i}=\beta_{0} x_{i}^{\beta_{1}}+e_{i}\right)$. Os parâmetros $\beta_{0}, \beta_{1}$ e $\beta_{2}$ foram estimados pelo método dos mínimos quadrados tendo sido feita previamente a linearização da função potência.

Determinou-se o tamanho amostral necessário para estimação de cada uma das nove equações de regressão ajustadas pela equação $\eta_{\mathbb{e}}=\left(t_{\alpha / 2}^{2} R Q M E^{2} / e^{2} \bar{Y}^{2}\right)$, em que: $\eta_{\mathbf{e}}$ é o tamanho da amostra para determinar a equação; $\bar{Y}$ é a média aritmética da AFO na 
amostra de $n=200$ folhas; $t_{\alpha / 2}$ é o valor crítico da distribuição † de Student, cuja área à direita é igual a $\alpha / 2$ com $(n-1)$ graus de liberdade, e com $1 \%$ de probabilidade de erro; RQME é raiz do quadrado médio do erro; e é o erro na estimativa da média, assumido como $5 \%$. A RQME é dada por RQME $=\sqrt{\sum_{i=1}^{n}\left(\hat{Y}_{i}-Y_{i}\right)^{2} / n}$, em que $\hat{Y}_{i}$ são os valores estimados e $Y_{i}$ os valores observados de área foliar.

Para cada modelo foi ajustada uma regressão linear simples $\left(\hat{Y}_{i}=\hat{\beta}_{0}+\hat{\beta}_{1} Y_{i}\right)$ da área foliar estimada pelo modelo (variável dependente) emfunção da área foliarobservada (variável independente). Foram testadas as hipóteses $H_{0}: \beta_{0}=0$ versus $H_{a}: \beta_{0} \neq 0$ e $H_{0}: \beta_{1}=1$ versus $H_{a}: \beta_{1} \neq 1$, por meio do teste $t$ de Student a $5 \%$ de probabilidade de erro. Determinouse também a raiz do quadrado médio do erro (RQME), o erro absoluto médio (EAM) e o índice d de Willmott (Willmott, 1981), sendo estes últimos dois dados por: EAM $=\sum_{i=1}^{n}\left|\hat{Y}_{i}-Y_{i}\right| / n$; $d=1-\left[\sum_{i=1}^{n}\left(\hat{Y}_{i}-Y_{i}\right)^{2} / \sum_{i=1}^{n}\left(\left|\hat{Y}_{i}-\bar{Y}\right|+\left|Y_{i}-\bar{Y}\right|\right)^{2}\right]$. Os resíduos obtidos entre a área foliar observada e estimada para cada modelo foram submetidos ao teste de Shapiro-Wilk para verificação da normalidade.

Os critérios utilizados para a seleção dos modelos que melhor estimam a área foliar em função de $C$, L ou CL, foram, com base nos parâmetros estimados: coeficiente linear $\left(\beta_{0}\right)$ não diferente de zero e coeficiente angular $\left(\beta_{1}\right)$ não diferente de um, indicando que a área foliar estimada pelo modelo é semelhante à área foliar observada; coeficiente de determinação $\left(R^{2}\right)$ mais próximo de um; erro absoluto médio (EAM) e raiz do quadrado médio do erro (RQME) mais próximo de zero; índice d mais próximo de um e; valor-p do teste de Shapiro-Wilk maior que 0,05 . As análises estatísticas foram realizadas com auxílio do aplicativo Microsoft Office Excel e do software Genes (Cruz, 2013).

\section{Resultados e Discussão}

Os resultados da análise de variância para medidas alométricas do limbo foliar, indicaram que não houve diferença estatística significativa, em nível de $5 \%$ de erro, entre as médias das variáveis na comparação entre plantas (Tabela 1). Com base neste resultado, a análise de modelagem estatística foi feita com uso conjunto das 200 folhas coletadas das cinco plantas.

A variabilidade, medida pelo coeficiente de variação foi em torno de $25 \%$ para C e para $\mathrm{L}$, cerca de $50 \%$ para $\mathrm{CL}$ e para área foliar observada (AFO) e de apenas 3\% para o fator $f$ (Tabela 1). Os altos valores observados para C, L, CL e AFO mostram que a amostra de folhas é bem representativa da população, com folhas de diferentes tamanhos, sendo interessante para trabalhos de modelagem, podendo as equações serem usadas para folhas pequenas, médias e grandes (Cargnelutti Filho et al., 2012). Por outro lado a baixa variabilidade encontrada para o fator $\mathrm{f}$ mostra que este fator não se altera em relação à área das folhas que variou de 4,66

Tabela 1. Análise de variância de medidas alométricas de comprimento $(C$, em $\mathrm{cm})$, maior largura $(\mathrm{L}$, em $\mathrm{cm})$, comprimento vezes maior largura $(\mathrm{CL})$ de limbo foliar, área foliar observada (AFO, em cm²) e fator de correção (f) em folhas de "pata de vaca" (Bauhinia monandra)

\begin{tabular}{|c|c|c|c|c|c|c|}
\hline \multirow{2}{*}{ Fonte de Variação } & \multirow{2}{*}{$\begin{array}{l}\text { Graus de } \\
\text { liberdade }\end{array}$} & \multicolumn{5}{|c|}{ Quadrado médio } \\
\hline & & C & $\mathrm{L}$ & $\mathrm{CL}$ & $\mathrm{AFO}$ & $f\left(x 10^{4}\right)$ \\
\hline Entre plantas & 4 & $1,77^{\mathrm{ns}}$ & $0,52 \mathrm{~ns}$ & $437,03^{\text {ns }}$ & $226,76^{\mathrm{ns}}$ & $4,27^{\mathrm{ns}}$ \\
\hline Dentro de plantas & 195 & 6,21 & 6,52 & 3026,65 & 1868,09 & 7,00 \\
\hline Mínimo & & 2,30 & 2,40 & 5,52 & 4,66 & 0,77 \\
\hline Máximo & & 18,10 & 19,80 & 349,20 & 271,17 & 0,92 \\
\hline Média & & 9,98 & 10,34 & 109,35 & 90,12 & 0,83 \\
\hline $\mathrm{CV}(\%)$ & & 24,96 & 24,69 & 50,31 & 47,96 & 3,19 \\
\hline
\end{tabular}

até $271,17 \mathrm{~cm}^{2}$.

$\mathrm{Na}$ obtenção das equações de estimativa da área foliar, os modelos apresentaram ajustes com elevados valores dos coeficientes de determinação $\left(R^{2} \geq 0,9432\right)$
(Tabela 2). O maior coeficiente de determinação $\left(R^{2}=0,9967\right)$ foi obtido para o modelo potencial a partir de CL. Resultados semelhantes de modelos potencial ajustados em função do produto entre comprimento e largura também 
foram encontrados para outras culturas, como nabo forrageiro (Cargnelutti Filho et al., 2012) e amendoim (Cardozo et al., 2014).

O tamanho amostral mínimo requerido para este estudo foi de 35 folhas (Tabela 2). Portanto, o tamanho de 200 folhas, usado neste trabalho dá credibilidade ao estudo com uma confiança de $99 \%$ e erro de $5 \%$ da média. $\mathrm{Na}$ literatura tem sido variável o número de folhas usadas na modelagem, onde são verificados desde 18 folhas para videira 'CabernetSauvignon' (Tsialtas et al., 2008) até 720 folhas para mangueira 'Tommy Atkins' (Lima et al., 2012).

Tabela 2. Equações ajustadas para a determinação da área foliar, em $\mathrm{cm}^{2}\left(\hat{Y}_{\mathrm{i}}\right)$, utilizando medidas alométricas do limbo foliar como variáveis independentes $\left(x_{i}\right)$, coeficiente de determinação $\left(R^{2}\right)$ e tamanho mínimo da amostra $\left(n_{\text {eq }}\right)$, com base em 200 folhas de "pata de vaca" (Bauhinia monandra)

\begin{tabular}{|c|c|c|c|c|}
\hline $\begin{array}{c}\text { Medida } \\
\text { alométrica(1) }^{(1)}\end{array}$ & $\begin{array}{c}\text { Modelo } \\
\text { matemático }\end{array}$ & Equações & $\mathrm{R}^{2}$ & $\mathrm{n}_{\mathrm{eq}}$ \\
\hline C & Linear & $\hat{Y}_{i}=-77,7887+16,78185 x_{i}$ & 0,9432 & 35 \\
\hline L & Linear & $\hat{Y}_{i}=-81,1091+16,5538 x_{i}$ & 0,9558 & 27 \\
\hline $\mathrm{CL}$ & Linear & $\hat{Y}_{i}=4,4751+0,7832 x_{i}$ & 0,9943 & 3 \\
\hline C & Quadrático & $\hat{Y}_{i}=0,1035+0,8521 x_{i}+0,7707 x_{i}^{2}$ & 0,9859 & 9 \\
\hline L & Quadrático & $\hat{Y}_{i}=-19,5721+4,7497 x_{i}+0,5342 x_{i}^{2}$ & 0,9827 & 10 \\
\hline $\mathrm{CL}$ & Quadrático & $\hat{Y}_{i}=-1,4937+0,8799 x_{i}-0,0004 x_{i}^{2}$ & 0,9961 & 4 \\
\hline C & Potencial & $\hat{Y}_{i}=1,0332 x_{i}^{1,9191}$ & 0,9886 & 9 \\
\hline L & Potencial & $\hat{Y}_{i}=0,8294 x_{i}^{1,9833}$ & 0,9859 & 16 \\
\hline$C L$ & Potencial & $\hat{Y}_{i}=0,8887 x_{i}^{0,9846}$ & 0,9967 & 4 \\
\hline
\end{tabular}

Na verificação da validação (Tabela 3), o modelo potencial em função de CL se mostrou inadequado, por apresentar coeficiente linear diferente de zero, apesar de ter apresentado o maior $\mathrm{R}^{2}$ na obtenção do modelo (Tabela 2). Pelo mesmo motivo, também se mostraram inadequados os modelos linear para C, quadrático para CL e potencial para L. Também é inadequado o modelo linear para L, por apresentar coeficiente angular diferente de um. Dentre os demais modelos, considerando o erro absoluto médio (EAM) e raiz do quadrado médio do erro (RQME) mais próximos de zero, coeficiente de determinação e índice $d$ de Willmott (Willmott, 1981) mais próximo de um, e com resíduos apresentando distribuição normal, o modelo mais apropriados é o linear $\hat{Y}_{i}=4,4751+0,7832(C L)$. Os modelos quadrático para $C$ e para $L$ e o potencial para C também se mostraram adequados.

Na prática, o uso de equações de modelos lineares baseados em apenas uma dimensão são preferíveis por sua simplicidade para aplicação, principalmente no campo (Tsialtas \& Maslaris, 2005). Neste caso, para o presente trabalho, indica-se a equação potencial baseado no comprimento do lóbulo foliar dada por $\hat{Y}_{i}=1,0332 \mathrm{C}_{i}^{1,9191}$ (Tabela 2) e 
Tabela 3. Coeficientes linear $\left(\beta_{0}\right)$, angular $\left(\beta_{1}\right)$, coeficiente de determinação $\left(R^{2}\right)$ obtidos na regressão ajustada entre a área foliar estimada (variável dependente) e a observada (variável independente), erro absoluto médio (EAM), raiz do quadrado médio do erro (RQME), índice d de Willmott (Willmott, 1981) e valor-p do teste de ShapiroWilk calculados pela diferença entre as áreas foliares estimadas e observadas de 55 folhas de "pata de vaca" (Bauhinia monandra)

\begin{tabular}{|c|c|c|c|c|c|c|c|c|}
\hline $\begin{array}{c}\text { Medida } \\
\text { alométrica }^{(1)}\end{array}$ & $\begin{array}{c}\text { Modelo } \\
\text { matemático }\end{array}$ & $\hat{\beta}_{0}^{(2)}$ & $\hat{\beta}_{1}^{(3)}$ & $\mathrm{R}^{2}$ & EAM & RQME & $d$ & Valor-p \\
\hline C & Linear & $8,0168^{*}$ & $0,9153^{* *}$ & 0,9562 & 8,0451 & 10,7081 & 0,9935 & $<0,01$ \\
\hline L & Linear & $4,5815^{\mathrm{ns}}$ & $0,9388^{* *}$ & 0,9717 & 6,5344 & 8,7396 & 0,9957 & 0,0369 \\
\hline$C L$ & Linear & $0,0768^{\text {ns }}$ & $1,0018^{\text {ns }}$ & 0,9941 & 2,8934 & 3,8617 & 0,9992 & 0,0686 \\
\hline C & Quadrático & $2,0179 \mathrm{~ns}$ & $0,9866^{\mathrm{ns}}$ & 0,9877 & 4,1159 & 5,5833 & 0,9983 & 0,0524 \\
\hline L & Quadrático & $0,4671^{\text {ns }}$ & $0,9869^{n s}$ & 0,9833 & 5,1963 & 6,5348 & 0,9977 & 0,9950 \\
\hline$C L$ & Quadrático & $2,0548^{*}$ & $0,9633^{* *}$ & 0,9968 & 2,7694 & 3,6811 & 0,9992 & 0,1059 \\
\hline C & Potencial & 1,4829 ns & $0,9923^{\mathrm{ns}}$ & 0,9876 & 4,1286 & 5,6195 & 0,9983 & 0,0732 \\
\hline L & Potencial & $-5,8383^{*}$ & $1,0594^{* *}$ & 0,9778 & 5,8274 & 8,5262 & 0,9962 & $<0,01$ \\
\hline $\mathrm{CL}$ & Potencial & $-3,1275^{*}$ & $1,0366^{* *}$ & 0,9946 & 2,6809 & 4,2834 & 0,9990 & $<0,01$ \\
\hline
\end{tabular}

com critérios aceitáveis de validação (Tabela 3).

\section{Agradecimentos}

Os autores agradecem ao CNPq e à

CAPES pelo auxílio financeiro por meio de bolsas.

\section{Referências}

Cardozo, N.P., Parreira, M.C., Panosso, A.R., Volpe, C.A. 2014. Modelagem da área foliar de duas cultivares de amendoim em função das dimensões lineares dos folíolos. Bioscience Journal 30:101-107.

Cargnelutti Filho, A., Toebe, M., Burin, C., Fick, A.L, Casarotto, G. 2012. Estimativa da área foliar de nabo forrageiro em função de dimensões foliares. Bragantia 71:47-51.

Collins, T.J. 2007. ImageJ for microscopy. BioTechniques 43:25-30.

Cruz, C.D. 2013. GENES: a software package for analysis in experimental statistics and quantitative genetics. Acta Scientiarum Agronomy 35:271276.

Demirsoy, H. 2009. Leaf area estimation in some species of fruit tree by using models as nondestructive method. Fruits 64:45-51.

Francisco, J.P., Diotto, A.V., Folegatti, M.V., Silva, L.D.B., Piedade, S.M.S. 2014. Estimativa da área foliar do abacaxizeiro cv. Vitória por meio de relações alométricas. Revista Brasileira de Fruticultura 36:285-293.

Lima, R.T., Souza, P.J.O.P., Rodrigues, J.C., Lima, M.J.A. 2012. Modelos para estimativa da área foliar da mangueira utilizando medidas lineares. Revista Brasileira de Fruticultura 34:974-980.

Lusa, M.G., Bona, C. 2009. Análise morfoanatômica comparativa da folha de
Bauhinia forficata Link e B. variegata Linn. (Leguminosae, Caesalpinioideae). Acta Botanica Brasilica 23:196-211.

Sarker, S.K., Das, N., Chowdhury, M.Q., Haque, M.M. 2013. Developing allometric equations for estimating leaf area and leaf biomass of Artocarpus chaplasha in Raghunandan Hill Reserve, Bangladesh. Southern Forests: a Journal of Forest Science 75:51-57.

Souza, M.S., Alves, S.S.V., Dombroskl, J.L.D., Freitas, J.D.B., Aroucha, E.M.M. 2012. Comparação de métodos de mensuração de área foliar para a cultura da melancia. Pesquisa Agropecuária Tropical 42:241-245.

Tsialtas J.T., Koundouras S., Zioziou E. 2008. Leaf area estimation by simple measurements and evaluation of leaf area prediction models in Cabernet-Sauvignon grapevine leaves. Photosynthetica 46:452-456.

Tsialtas J.T., Maslaris N. 2005. Leaf area estimation in a sugar beet cultivar by linear models. Photosynthetica 43:477-479.

Vaz, A.M.S.F., Tozzi, A.M.G.A. 2005. Sinopse de Bauhinia sect. Pauletia (Cav.) D.C. (Leguminosae: Caesalpinoideae: Cercideae) no Brasil. Revista Brasileira de Botânica 28:477-491.

Willmott, C.J. 1981. On the validation of models. Physical Geography 2:184-194. 